\title{
Molecular Conformation and Hydrogen Bond Formation in Liquid Ethylene Glycol
}

\author{
Aman Jindal and Sukumaran Vasudevan* \\ Department of Inorganic and Physical Chemistry \\ Indian Institute of Science, Bangalore 560012, \\ India
}

Supporting Information

*Author to whom correspondence may be addressed. E-mail: svipc@iisc.ac.in. Tel: +91-802293-2661. Fax: +91-80-2360-1552/0683; 


\section{CONTENTS}

S1) Determining the ${ }^{1} \mathrm{H}$ NMR distance ratio: $r_{\mathrm{CH}_{2} \cdots \mathrm{OH}}^{\text {inter }} / r_{\mathrm{CH}_{2} \cdots \mathrm{OH}}^{\text {intra }}$

S2) Identifying outliers: The Mahalanobis distance criteria.

S3) Plots of $P_{r e f}(r, \theta)$ for GG and GT(TG) pairs. 
S1. Determining the ${ }^{1} \mathrm{H}$ NMR distance ratio: $r_{\mathrm{CH}_{2} \cdots O H}^{i n t e r} / r_{\mathrm{CH}_{2} \cdots O H}^{i n t r a}$

The NMR distance ratio $r_{\mathrm{CH}_{2} \cdots \mathrm{OH}}^{\text {inter }} / r_{\mathrm{CH}_{2} \cdots \mathrm{OH}}^{\mathrm{intra}}$ was determined from the ${ }^{\mathrm{I}} \mathrm{H}$ nuclear Overhauser effect (NOE) NMR measurements on a diluted mixture of $\mathrm{HOCH}_{2} \mathrm{CH}_{2} \mathrm{OH}$ (I) and deuterated $\mathrm{HOCD}_{2} \mathrm{CD}_{2} \mathrm{OH}(\mathbf{I I})$ in $\mathrm{CDCl}_{3}$ (Figure $\mathrm{S} 1$ ), reported in Reference 30. The two distinct resonances at 2.82 and $2.78 \mathrm{ppm}$ in Figure S1 correspond to the hydroxyl resonances of molecules I and II, respectively; the difference in chemical shifts arising from the secondary isotope effect. The resonance at $3.7 \mathrm{ppm}$ are the methyl protons of $\mathbf{I}$, these are obviously absent in II. NOE was used to distinguish intra- and intermolecular distances in the mixture by exploiting the fact that NOE cross-relaxation between two nuclei is directly related to the interproton distance.

Within the initial rate approximation, the NOE enhancement, $\eta_{I}\{S\}$, of spin I on inversion or irradiation of $\operatorname{spin} \mathrm{S}$, at a mixing time of $\tau_{m}$ is proportional to the cross relaxation rate, $\sigma_{I S}$, which in turn depends on their internuclear separation, $r_{I S}^{-6}$, and is given by the expression

$$
\eta_{I}\{S\}=k \sigma_{I S} \tau_{m}=k^{\prime} r_{I S}^{-6} \tau_{m}
$$

where $k$ and $k^{\prime}$ are constants of proportionality. In the linear regime the ratio for a pair of NOE signals, $\eta_{I 1}\{S\}, \eta_{I 2}\{S\}$ for spins $\mathrm{I}_{1}$ and $\mathrm{I}_{2}$ would be proportional to the ratio of the internuclear distances between these spins and the spin S, that is undergoing inversion, and will be independent of mixing time.

$$
\frac{\eta_{I 2}\{S\}}{\eta_{I 1}\{S\}}=\frac{\sigma_{I 2 S}}{\sigma_{I 1 S}}=\frac{r_{I 2 S}^{-6}}{r_{I 1 S}^{-6}}
$$




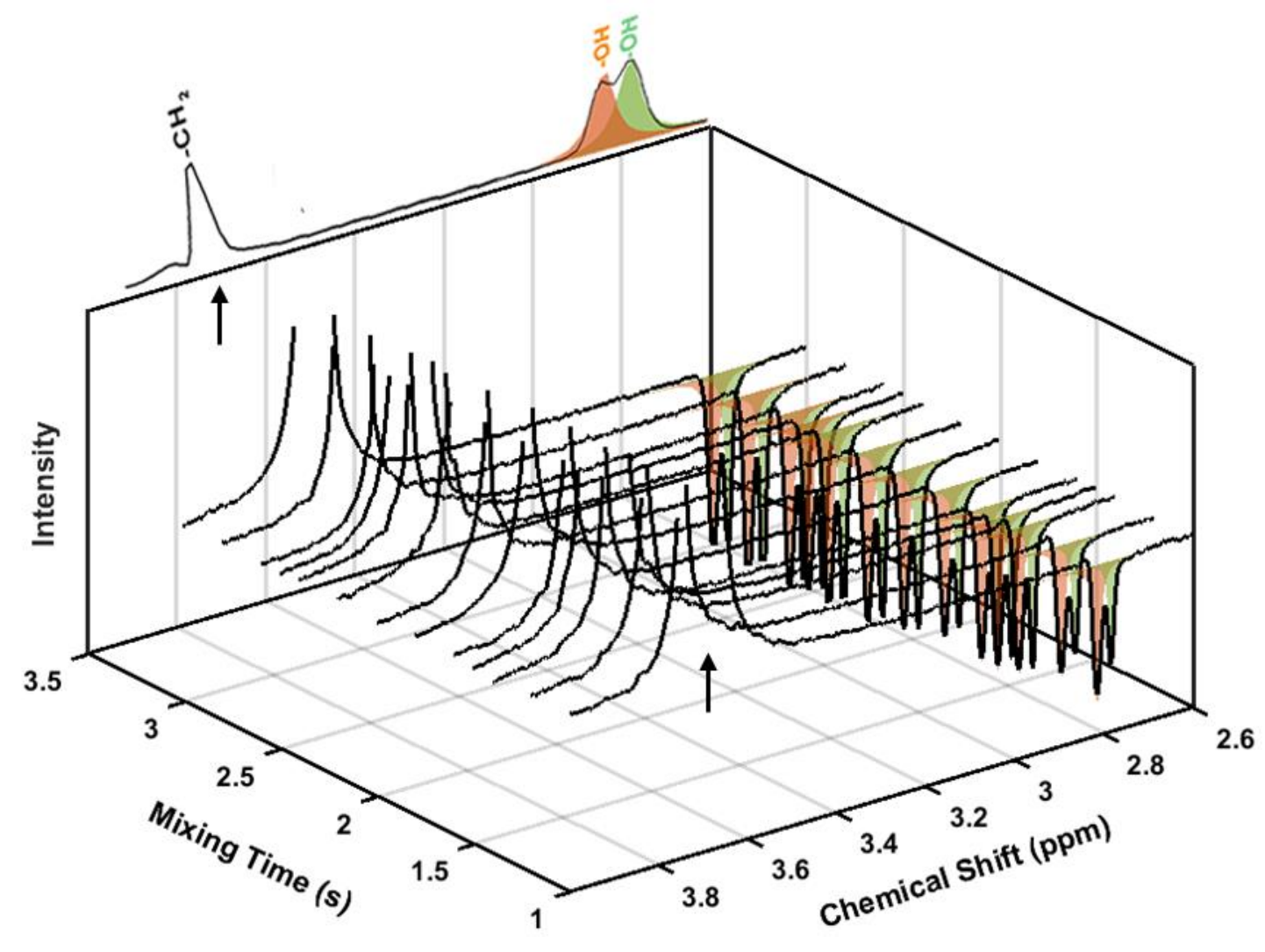

Figure S1. The ${ }^{1} \mathrm{H}$ NMR spectra of a 1:1 diluted mixture of $\mathrm{HOCH}_{2} \mathrm{CH}_{2} \mathrm{OH}$, and deuterated $\mathrm{HOCD}_{2} \mathrm{CD}_{2} \mathrm{OH}$ (mole fraction 0.03) at different mixing times on irradiation of the $\mathrm{CH}_{2}$ peak at $3.7 \mathrm{ppm}$ (shown by arrow). Filled peaks represent the hydroxyl resonances of $\mathrm{HOCH}_{2} \mathrm{CH}_{2} \mathrm{OH}$ (orange), and deuterated $\mathrm{HOCD}_{2} \mathrm{CD}_{2} \mathrm{OH}$ (green). (adapted from Reference 30)

The 1D transient NOE experiments on the diluted 1:1 mixture of I and II reported in Reference 30 had been performed by selective inversion of the resonance at $3.7 \mathrm{ppm}$, due to the $\mathrm{CH}_{2}$ protons of I, and measuring the intensities of the two hydroxyl resonances at $2.82 \mathrm{ppm}$, due to the $\mathrm{OH}$ protons of I, and $2.78 \mathrm{ppm}$, the $\mathrm{OH}$ protons of II, at different mixing times (Figure S1). The intensity variation of the two resonances with mixing time, following inversion, would reflect the 
intra- and intermolecular distances between the $\mathrm{CH}_{2}$ and $\mathrm{OH}$ protons, respectively. The intensities of the hydroxyl resonances in the mixture were obtained after decomposing the hydroxyl resonances as a sum of two Lorentzians; centered at 2.82 and $2.78 \mathrm{ppm}$. The intensity of the $-\mathrm{OH}$ resonance of I was obtained by integrating the Lorentzian at $2.82 \mathrm{ppm}$ while that of II from the integration of the resonance at $2.78 \mathrm{ppm}$. The ratio of the intensities of $\mathrm{OH}$ resonances of II and I at different mixing times is plotted Figure S2. The ratio is independent of the mixing time and has a value of 1.03 . This value may be directly related to the ratio of the intermolecular distance between the $\mathrm{CH}_{2}$ protons and the $\mathrm{OH}$ protons with the corresponding intramolecular distance , $r_{\mathrm{CH}_{2} \cdots \mathrm{OH}}^{\text {inter }} / r_{\mathrm{CH}_{2} \cdots \mathrm{OH}}^{\mathrm{intra}}$, using eq. 2 ; the value is 1.005 .

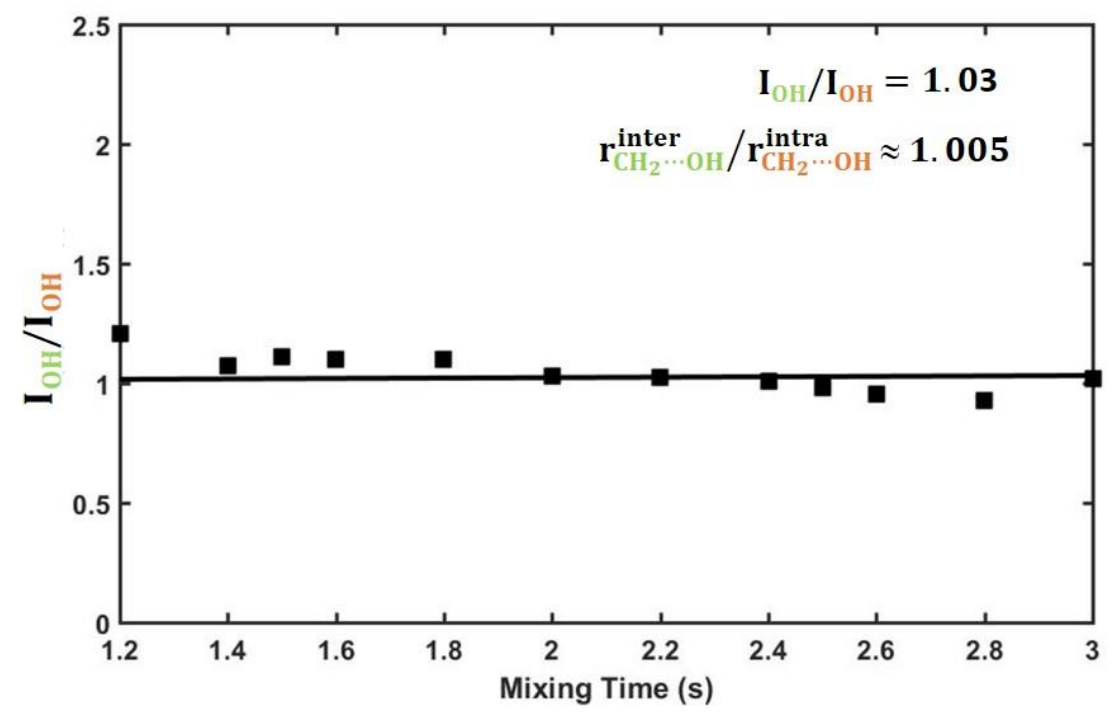

Figure S2. Variation of the intensity ratio for $\mathrm{OH}$ resonances at $2.82\left(\mathrm{HOCD}_{2} \mathrm{CD}_{2} \mathrm{OH}\right)$ and 2.78 ppm $\left(\mathrm{HOCH}_{2} \mathrm{CH}_{2} \mathrm{OH}\right)$ shown in Figure $\mathrm{S} 1$ with mixing time. 


\section{S2. Identifying outliers: The Mahalanobis distance criteria.}

. The Mahalanobis distance criteria was used to identify the outliers in the scatter plots of Figures $3 \mathrm{~b}$ and $3 \mathrm{~d}$ and hence to define the cut-off values for the geometrical parameters for the intermolecular $\mathrm{OH} \cdots \mathrm{O}$ hydrogen bonds in liquid ethylene glycol

The Mahalanobis distance is a measure of the distance between a point $\mathrm{P}$ and a distribution $\mathrm{D}$, and is a well-established procedure to estimate the probability that a test point in $\mathrm{N}$-dimensional Euclidean space belongs to a set of observations. The Mahalanobis distance of an observation $\vec{x}$ from a set of observations with mean $\vec{\mu}$ and covariance matrix $\mathrm{S}$ is defined as:

$$
D_{M}(\vec{x})=\sqrt{(\vec{x}-\vec{\mu}) S^{-1}(\vec{x}-\vec{\mu})}
$$

We chose the Mahalanobis distance rather than the Euclidean distance as the former is unit-less and scale-invariant. It was observed that for EG pairs satisfying the NMR distance ratio criteria, the values of the $\boldsymbol{r}_{\mathrm{OH} \cdots \mathrm{O}}$ and $\boldsymbol{r}_{\mathrm{O} \cdots \mathrm{O}}$ distances and $\angle \mathrm{HO} \cdots \mathrm{O}(\theta)$ angle followed a Gaussian distribution (FigureS3) both for GG and GT(TG) pairs and therefore we computed the squared Mahalanobis distance (Equation S3) and compared the value to the square root of $\chi^{2}(3,0.975) \approx$ 3.05, which is the $97.5 \%$ quantile of the chi-square distribution with three degrees of freedom. The points whose squared Mahalanobis distance value exceed 3.05 were identified as outliers. In Figure S4 the squared Mahalanobis distance for each of the points on Figures $3 \mathrm{~b}$ and $3 \mathrm{~d}$ have been plotted ;the outliers are shown in red. 

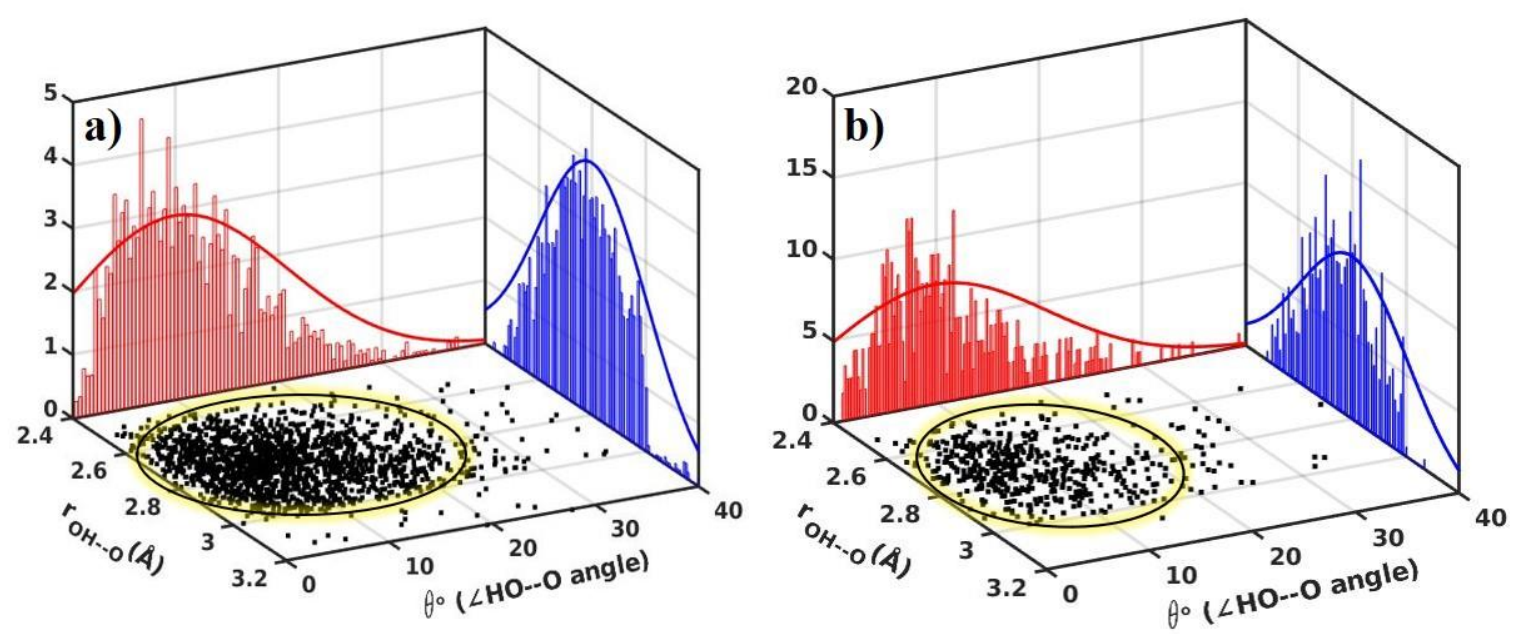

Figure S3. The distribution of non-bonded parameters, $\boldsymbol{r}_{\mathrm{OH} \cdots \mathrm{O}}(\AA)$ and $\theta^{\circ}(\angle \mathrm{HO} \cdots \mathrm{O})$ for data points with conformers a) GG b) GT(TG) satisfying the NMR distance ratio criteria, $r_{\mathrm{CH}_{2} \cdots \mathrm{OH}}^{\text {inter }} / r_{\mathrm{CH}_{2} \cdots \mathrm{OH}}^{\text {intra }}=1.005$. The red and blue lines are the Gaussian profiles.
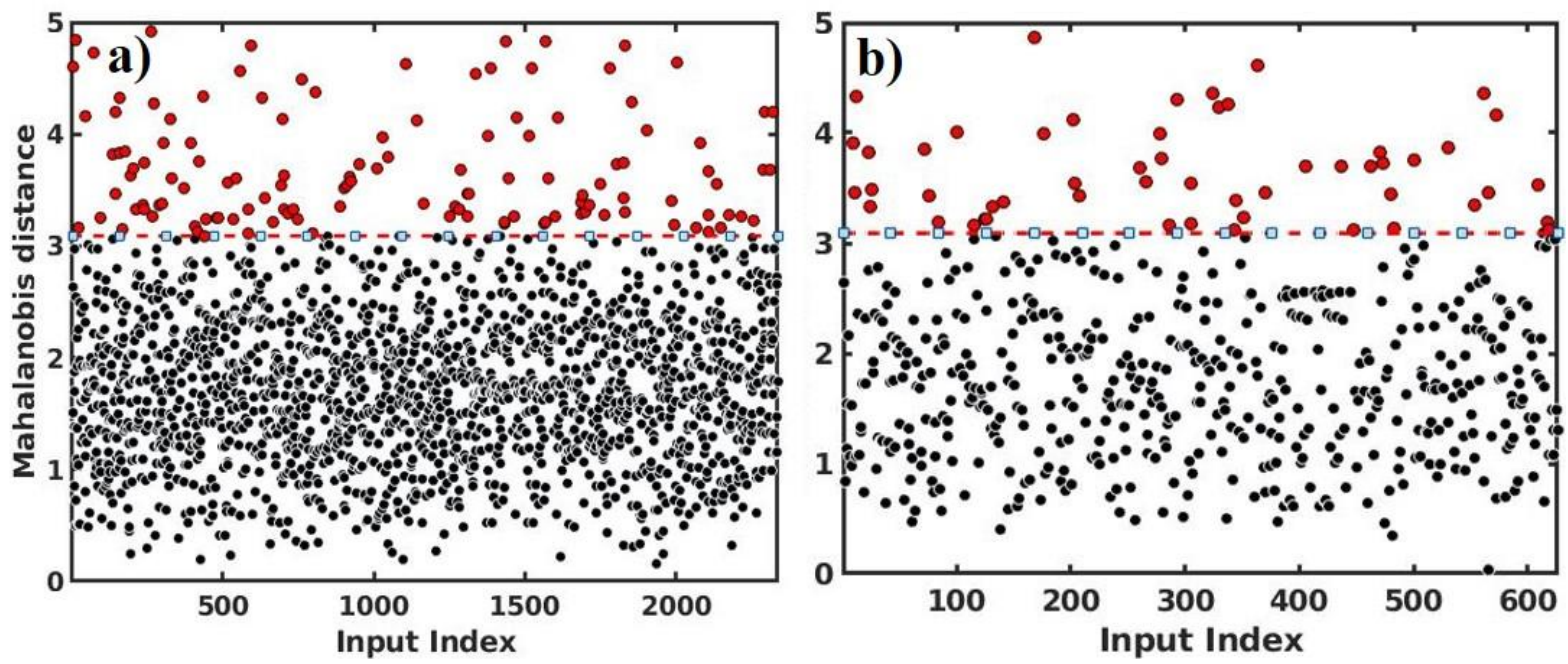

Figure S4. The squared Mahalanobis distance plot for a) GG and b) GT(TG) pairs. The dashed line corresponds to the cut-off value, $\sqrt{\chi_{3,0.975}^{2}}=3.05$. The outliers are shown in red. 
S3. Plots of $\boldsymbol{P}_{r e f}(\boldsymbol{r}, \boldsymbol{\theta})$ for GG and GT(TG) pairs.
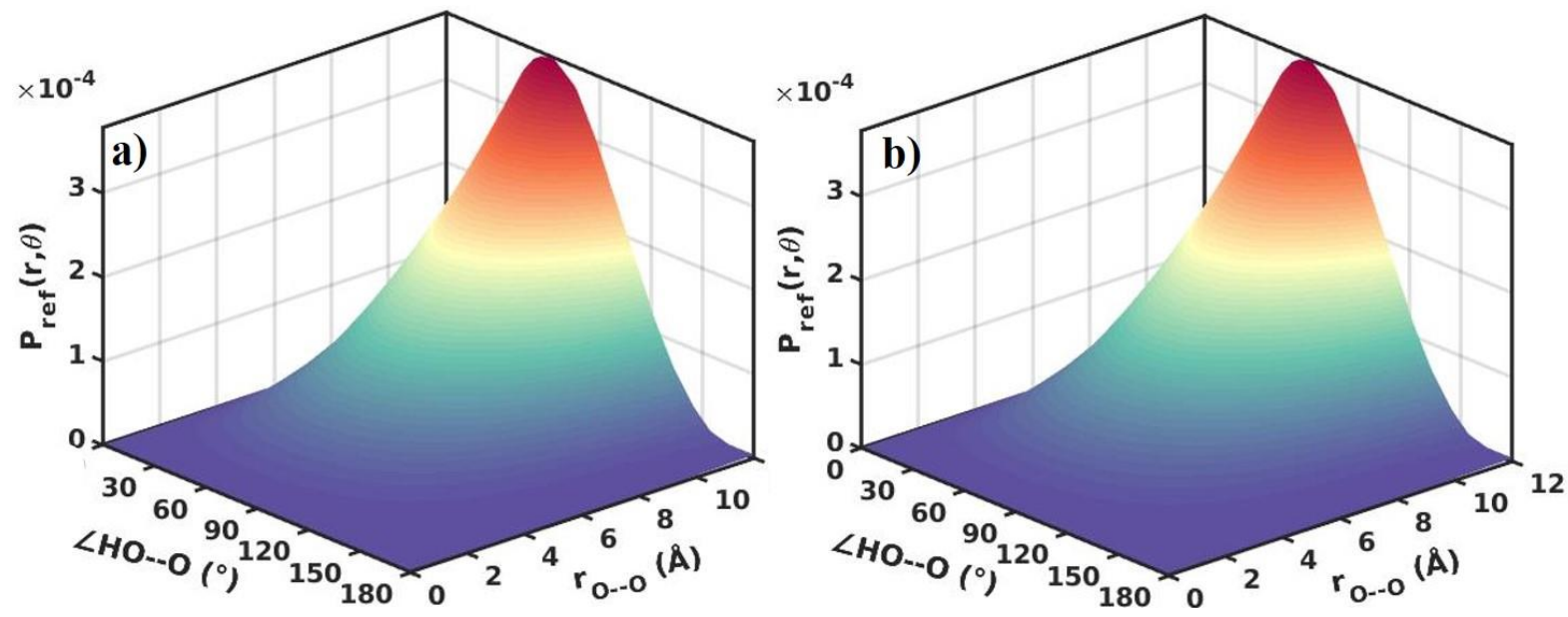

Figure S5. The reference probability density distributions, $P_{r e f}(r, \theta)$ for a) GG and b) GT(TG) pairs. 